\title{
TENDÊNCIAS DE PADRONIZAÇÃO DE ARQUITETURAS E PROTOCOLOS DE REDES ÓPTICAS
}

\author{
Eduardo José Aloia, Amílcar Careli César e Murilo Araújo Romero
}

\begin{abstract}
Resumo - O objetivo deste trabalho é analisar as tendências de evolução das arquiteturas de redes ópticas, discutir o estado-da-arte dos protocolos necessários para seu desenvolvimento e avaliar os esforços que estão sendo realizados por diversas instituições como ITU (International Telecommunications Union), IETF (Internet Engineering Task Force) e OIF (Optical Internetworking Forum) para alcançar a padronização. As redes ópticas têm origem no início dos anos oitenta com o uso de fibra óptica monomodo, evoluindo com a introdução de uma nova base tecnológica, composta por novos tipos de fibras ópticas, amplificadores ópticos, sistemas DWDM (dense wavelength division multiplexing) e componentes como add/drop multiplexers e cross-connects ópticos. A introdução destes componentes tornará possível a evolução dos sistemas WDM ponto a ponto para as redes totalmente ópticas. As formas como as aplicações interagem com a camada física são analisadas neste artigo. Tal interação tem provado ser um desafio para as arquiteturas atuais de redes, as quais precisam dispor de soluções que permitam o transporte eficiente de grande volume de tráfego. Com este objetivo em foco, o uso de tecnologias como o MPLS (multiprotocol label switching) e sua evolução, o MPLS generalizado (GMPLS) é discutido. Finalmente, a versão em desenvolvimento do ITU para arquitetura de redes ópticas permitindo a inclusão do GMPLS é apresentada.
\end{abstract}

Palavras-chave: redes ópticas; arquitetura de redes; protocolos; padronização; internet protocol (IP); dense wavelength division multiplexing (DWDM); IP sobre WDM; MPLS (multiprotocol label switching); GMPLS (generalized MPLS).

Abstract - The main objective of this work is to analyze the
evolution trends for the architecture of optical networks,
discuss the state of the art for the protocols required for its
development and evaluate the various efforts being carried
out by different institutions as the ITU (International
Telecommunications Union), IETF (Internet Engineering
Task Force) and OIF (Optical Internetworking Forum) in
order to achieve standardization. Optical networks arose in
the early eighties with the use of the monomode fiber optic
cables and were further developed with the introduction of a
new technological base composed by new types of optical
fibers, optical amplifiers, DWDM (dense wavelength
division multiplexing) systems and optical components such
as add/drop multiplexers and optical cross-connects. The
introduction of these components will make possible the
evolution from the simple point-to-point WDM connections
to the all optical networks. In this work we analyze how the

Eduardo José Aloia, Amúlcar Careli César e Murilo Araújo Romero são da Universidade de São Paulo, Escola de Engenharia de São Carlos, Departamento de Engenharia Elétrica, Avenida Trabalhador São-carlense, 400, 13566-590 São Carlos, SP. Emails: \{eduja:amilcar;muriloa\}@sel.eesc.usp.br. applications will interact with this physical layer. Such interaction has been demonstrated to be a challenge within the framework of the current architecture of data networks, aiming at offering solutions for the transport of a great volume of traffic in an efficient manner. With this goal in mind, the use of technologies such as MPLS (multiprotocol label switching) and its evolution, the generalized MPLS (GMPLS) is discussed. Finally, we present the in progress ITU version for the architecture optical networks, addressing its merging with the GMPLS.

Keywords: optical network; network architecture; protocols; standardization; Internet protocol (IP): dense wavelength division multiplexing (DWDM); IP over WDM; MPLS (multiprotocol label switching); GMPLS (generalized MPLS).

\section{INTRODUÇÃO}

A consolidação da Internet como rede mundial tem estimulado o desenvolvimento de inúmeras aplicações, com destaque para aquelas que requerem ampla largura de faixa, como multimídia. Serviços tradicionais de telecomunicações, como a telefonia, também têm evoluído para operar de acordo com o padrão IP (internet protocol), evitando a duplicação de recursos de infra-estrutura em locais onde as redes de computadores estão implantadas (voz sobre IP). A disseminação da rede de serviços sem fio também está voltada para o acesso à Internet via dispositivos portáteis. Assim, o sucesso da rede mundial de computadores estabeleceu um modelo para todas as redes de telecomunicações, segundo qual qualquer que seja o serviço oferecido por elas, a meta tem sido permitir que o usuário tenha acesso às aplicações disponiveis na Internet. Entretanto, o aumento progressivo do número de usuários e a demanda por garantia de largura de faixa e qualidade de serviço têm exercido enorme pressão sobre a infra-estrutura das redes de telecomunicações. A partir desta demanda, a gerência das redes passou a lidar com compromissos entre largura de faixa, engenharia de tráfego, proteção contra falhas e expansão da planta instalada, com intuito de garantir o melhor desempenho possível e plena satisfação do cliente. $\mathrm{O}$ atendimento desta demanda crescente tem sido possível graças às redes ópticas que fazem uso da multiplexação por divisão em comprimento de onda (WDM-wavelength division multiplexing), técnica capaz de aproveitar eficientemente a ampla largura de faixa da fibra óptica. Por este motivo, as redes ópticas passaram a ser a opção preferencial de infra-estrutura de transporte de grandes volumes de tráfego de informaçōes.

A expansão da infra-estrutura das redes de telecomunicações tem gerado a necessidade imperiosa de padronizar os sistemas de transmissão, porque as várias operadoras de serviços locais, cada uma com um sistema 
próprio, têm necessidade de se conectar às de longa distância. Isto ocorreu particularmente nos Estados Unidos e após a divisão da AT\&T, em 1984 [1]. Surgiu, então, o padrão SONET/SDH (synchronous optical network/synchronous digital hierarchy), baseado na multiplexação por divisão em tempo (TDM-time division multiplexing) [2], [3]. A largura de faixa da fibra era dedicada somente a um canal, alocando janelas temporais para os diversos subcanais e configurando um sistema síncrono, controlado por um relógio mestre. Esta padronização permitiu ampla capacidade de operação entre os sistemas SONET/SDH comercializados por diferentes fornecedores e, consequientemente, a rápida disseminação da tecnologia.

A partir dos anos 90, as redes ópticas evoluíram de sistemas utilizando apenas um comprimento de onda para aqueles do tipo WDM. O esquema WDM combina vários comprimentos de onda em uma única fibra óptica. Cada comprimento de onda pode transportar sinais com diferentes taxas de transmissão (2,5 e 10 Gbps, por exemplo) e formatos distintos. Os sistemas atuais operam em torno de $1550 \mathrm{~nm}$. Recentemente, a denominação DWDM (dense WDM) foi criada para distinguir os atuais sistemas WDM, com 0,8 nm (aproximadamente $100 \mathrm{GHz}$ ), ou menos, de espaçamento entre canais, dos pioneiros, que empregavam apenas dois comprimentos de onda (1310 e $1550 \mathrm{~nm})$.

A introdução gradativa de novos tipos de dispositivos ópticos, como amplificadores, multiplexadores $\mathrm{e}$ demultiplexadores add-drop (OADM-optical add-drop multiplexer) e cross-connects ópticos (OXC-optical crossconnect), tem possibilitado a ampliação do atendimento da demanda e permitirá nova etapa de evolução, agora em direção à rede totalmente óptica. Uma vez que a maior parte das soluções disponíveis, incluindo óptica integrada, não consegue atender adequadamente a demanda por um aumento na dimensionalidade da matriz de comutação, a utilização de sistemas microeletromecânicos (MEMSmicro-electromechanical systems) [4] tem sido a tendência atual na implementação de OADMs e OXCs. Embora ressalvada a sua limitação de velocidade de comutação, que pode se tornar um problema com o progressivo aumento do dinamismo na alocação de comprimentos de onda, o emprego destes dispositivos vem estimulando a proposição de novos padrões de sistemas de transmissão e arquiteturas de redes ópticas, nas quais funções como comutação e roteamento e inserção e retirada de canais, tradicionalmente realizadas no domínio eletrônico, passem a ser realizadas no domínio óptico. Adicionalmente, a tendência de compatibilidade com IP exige a simplificação das arquiteturas multicamadas para proporcionar operabilidade entre os diversos protocolos com finalidade de tornar as redes mais dinâmicas e eficientes. Um protocolo/arquitetura de rede tem se destacado para integrar as aplicações IP e as redes de transporte: é o MPLS (multiprotocol label switching) e sua extensão, o GMPLS (generalized multiprotocol label switching).

No sistema convencional de roteamento IP, o conteúdo do cabeçalho de um pacote é examinado para que a próxima posição no caminho fonte-destino seja determinada, tornando 0 processo demorado e ineficiente em ambiente de tráfego intenso. Por outro lado, os protocolos
MPLS/GMPLS são baseados em rótulos com significado local, examinados apenas pelos dispositivos diretamente envolvidos, tornando o roteamento mais eficiente.

Portanto, o MPLS é um protocolo cuja finalidade é proporcionar gerenciamento eficiente de fluxo de tráfego através da rede. O MPLS mapeia endereços IP em rótulos de tamanho fixo utilizados por tecnologias de comutação de pacotes, permite aproveitamento de protocolos consagrados, como o RSVP (resource reservation protocol) e OSPF (open shortest path first), além de suportar protocolos ATM e frame relay [5], [6].

Por sua vez, o protocolo GMPLS estende a versatilidade do MPLS, proporcionando controle sobre dispositivos capazes de comutar não só pacotes, mas também intervalos de tempo, comprimentos de onda e fibras ópticas. O plano de controle comum permite simplificar a operação da rede e habilitar a QoS desejada para as novas aplicações [7]. Assim, credencia-se para utilização em redes ópticas.

Há três organismos desenvolvendo padrões para redes ópticas: o ITU Telecommunication Standardization Sector (ITU-T), que é um setor do International Telecommunication Union (ITU) [8], Optical Internetworking Forum (OIF) [9] e The Internet Engineering Task Force (IETF) [10]. O OIF e IETF padronizam o plano de controle para as redes ópticas, enquanto o ITU-T, por intermédio dos grupos de estudos 13 e 15, desenvolve padrões para o plano de controle e arquitetura completa de rede óptica. $O$ conceito de rede óptica, ou ASON (automatically switched optical networks), como é denominada no âmbito do ITU-T, foi proposto com intuito de transportar vários "clientes", como SONET/SDH, Ethernet e ATM, em redes WDM, sob gerenciamento unificado. Os protocolos MPLS/GMPLS estão sendo padronizados pelo IETF.

No Brasil, também há iniciativas para investigar tais tipos de arquiteturas de rede. Uma delas é a parceria entre a Fundação Centro de Pesquisa e Desenvolvimento em Telecomunicações (CPqD) [11] e a Rede Nacional de Ensino e Pesquisa (RNP) [12], com recursos financeiros do Fundo para o Desenvolvimento Tecnológico das Telecomunicações (FUNTTEL) [13] e apoio da Financiadora de Estudos e Projetos (FINEP) [14], responsável pela proposição, desenvolvimento e gerência do projeto GIGA [15]. As metas deste projeto são desenvolver tecnologias de redes e aplicações voltadas para IP sobre WDM, capacitar empresas nacionais em tecnologias competitivas, em parceria com instituições de pesquisa e desenvolvimento (P\&D), além de fomentar a oferta de novos produtos, protocolos e serviços. O principal laboratório de testes é uma rede experimental de faixa larga-rede GIGA, interligando numa primeira fase instituições de P\&D nas cidades de Campinas, São Paulo, São José dos Campos, Cachoeira Paulista, Rio de Janeiro e Petrópolis. A outra iniciativa é o Programa Tecnologia de Informação no Desenvolvimento da Internet Avançada (TIDIA) [16], financiado pela FAPESP, com a participação de várias instituições de ensino e pesquisa. A finalidade do programa é promover avanço significativo em quantidade e qualidade nas pesquisas cooperativas em todas as áreas, desenvolvendo o sistema de pesquisa e inovação no Estado de São Paulo. Dentre os projetos que fazem parte deste programa destaca-se o Projeto KyaTera [17], cujo objetivo 
é estabelecer uma rede de fibras ópticas interligando laboratórios para estudo, pesquisa, desenvolvimento $\mathrm{e}$ demonstração de tecnologias $\mathrm{e}$ aplicações da Internet Avançada, permitindo que laboratórios geograficamente distribuídos testem novas tecnologias.

Vários autores têm investigado as arquiteturas para as futuras redes integrando IP com WDM, a operabilidade entre os diversos protocolos e as tendências tecnológicas. De modo geral, os artigos já publicados enfatizam um aspecto tecnológico [18]-[20].

O objetivo deste artigo é avaliar a evolução das redes ópticas, analisar as limitações de desempenho devido à inserção de cabeçalhos das principais arquiteturas multicamadas, descrever os protocolos MPLS e GMPLS, discutir suas especificaçōes de forma unificada, e avaliar a possibilidade da inserção do GMPLS na arquitetura padronizada pelo ITU-T. Versão ampliada, porém menos atual, deste estudo está disponível em [21].

$O$ artigo está organizado da seguinte forma. A grade de frequiências para aplicações WDM está descrita na Seção 2; os fundamentos e evolução das arquiteturas de redes são apresentados na Seção 3; as limitações de desempenho das principais arquiteturas multicamadas devido à inserção de cabeçalhos superpostos são discutidas na Seção 4; a arquitetura MPLS e implementação de engenharia de tráfego em redes IP estão apresentadas na Seção 5; a arquitetura GMPLS está descrita na Seção 6; a padronização de redes ópticas no âmbito do ITU-T e a inserção da arquitetura GMPLS são discutidas na Seção 7. As conclusões são resumidas na Seção 8. O Apêndice reúne tabelas com as recomendações do ITU-T.

\section{GRADE DE FREQÜÊNCIAS PARA APLICAÇÕES WDM}

Em 2002, o ITU-T definiu a grade de freqüências e espaçamento entre canais para sistemas ópticos empregando WDM por intermédio da recomendação ITU-T G.694.1 [22]. A frequiência de referência da grade é 193,1 THz $(1552,52 \mathrm{~nm})$ e, segundo esta recomendação, se um sistema óptico utiliza $100 \mathrm{GHz}$ de espaçamento entre canais, as freqüências são definidas por $(193,1 \pm \imath \times 0,1) \mathrm{THz}$, na qual $n=0,1,2, \ldots$ O emprego do WDM em arquiteturas de redes é discutido na próxima seção.

\section{FUNDAMENTOS E EVOLUÇÃO DAS REDES}

A arquitetura de uma rede pode ser entendida como a descrição dos componentes que a integram, suas funções e inter-relações [3]. O protocolo é um conjunto de regras que disciplinam a comunicação entre equipamentos e garante que as transferências de informação sejam ordenadas e isentas de erros. Os diversos protocolos existentes são organizados em camadas, cada uma delas com uma ou mais funções específicas, como aplicação, comutação, roteamento e transporte. A topologia de uma rede diz respeito à maneira como os diversos nós estão interligados. Em redes ópticas, um ou mais comprimentos de onda são utilizados para interligar os nós, formando um canal unidirecional. A topologia física consiste de î̀nterligações entre dispositivos ópticos e fibras, enquanto a topologia virtual é formada pelos vários canais unidirecionais, pelos quais fluem as unidades de dados entre os nós fonte e destino [23].

Além do padrão SONET/SDH, os protocolos ATM (asynchronous transfer mode) e Gigabit Ethernet (GbE) também têm sido empregados em redes, compondo arquiteturas multicamadas.

A tecnologia ATM, que proporciona a implementação de redes orientadas à conexão, começou a ser desenvolvida e testada nos anos 70 e 80 [24]. O ATM utiliza células de tamanho constante, independentemente da informação transportada, e a conexão é estabelecida sob a forma de canais e rotas virtuais. A arquitetura original da RDSI logo se mostrou ineficiente para adaptar, com baixo custo, serviços com taxas de transmissão diferentes e grandes volumes de tráfego. As vantagens do ATM, como cabeçalho simples, capacidade de comutação dos nós (podendo operar desde taxas de $100 \mathrm{Mbps}, 2,5 \mathrm{Gbps}$ e até $10 \mathrm{Gbps}$ ), de suporte a várias classes de serviços e de garantia de qualidade de serviço (QoS- quality of service), o habilitaram a integrar diversas arquiteturas de redes de telecomunicações [3], [24].

Uma das arquiteturas mais utilizadas apresenta, portanto, quatro camadas de protocolos, IP/ATM/SONETSDH/WDM, conforme ilustra a Figura 1.a. Neste contexto, a camada IP é utilizada como camada de serviço, ATM para engenharia de tráfego, SONET/SDH para monitoramento, confiabilidade e restauração, e WDM para o transporte. No entanto, esta arquitetura vem se mostrando redundante e incapaz de permitir o transporte de grandes volumes de tráfego com custo adequado.

Em geral, arquiteturas multicamadas são suscetíveis a efeitos nos quais uma camada pode limitar a capacidade de expansão (scalability) de redes inteiras, tanto quanto aumentar seus custos. Com o objetivo de reduzir o número de camadas, a tendência dominante aponta para a seguinte evolução: a função de engenharia de tráfego, executada pela camada ATM, será absorvida pela camada IP; a capacidade de proteção, roteamento e comutação do protocolo SONET/SDH deverá ser absorvidas pela camada óptica. A arquitetura resultante será formada por apenas duas camadas, denominada IP sobre WDM, melhorando sensivelmente o desempenho global da rede.

A primeira parte deste objetivo pode ser alcançada por meio da inclusão na camada IP de novas funcionalidades proporcionadas pela tecnologia MPLS [6], conforme ilustra a Figura 1.b. A funcionalidade restante pode ser alcançada à medida que comutadores ópticos puderem ser acrescentados aos sistemas WDM (Figura 1.c), dotando esta camada de meios para realizar a comutação óptica. A arquitetura com duas camadas, ilustrada na Figura 1.d, surgirá a partir da utilização do GMPLS e da disponibilidade de sistemas WDM com comutação óptica.

Uma forma de comparar o desempenho das várias arquiteturas multicamadas é estimar a penalidade associada à quantidade de bytes que compõem o cabeçalho. Esta estimativa é feita na próxima seção. 


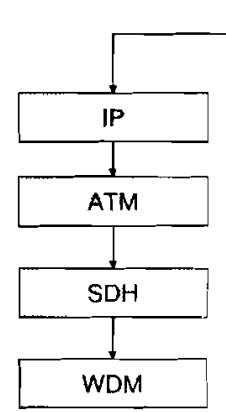

(a)

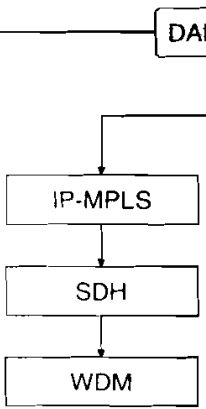

(b)

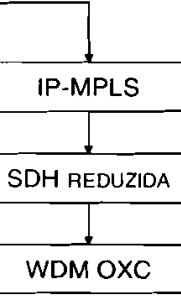

(c)

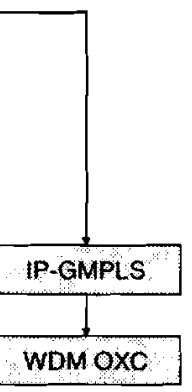

(d)
Figura 1. Evolução em direção à arquitetura de redes ópticas com duas camadas. IP: internet protocol; ATM: asynchromu, manster mode: SONET: synchronous optical network hicurch: WDM: wavelength division multiplexins: MIPLS: multiprotocol label switching; GMPLS: sencrulied multiprotocol label switching; OXC: optical charmermect.

\section{LIMITACÕ̃ES DE DESEMPENHO DAS PRINCIPAIS ARQUITETURAS MULTICAMADAS DEVIDO À INSERÇÃO DE CABEÇALHOS SUPERPOSTOS}

A Figura 2 mosula algumas formas de habilitar IP em redes óplicar IIDM. Cada uma delas provê diferentes funcionalidatc's 'll termos de capacidade de expansão, cabeçalho. İerenciamento de tráfego e qualidade de serviço. A quanticlade de bytes que cada protocolo utiliza para compor a calcecalho e a correspondente forma de processamento delerminam em grande parte a sua eficiência.

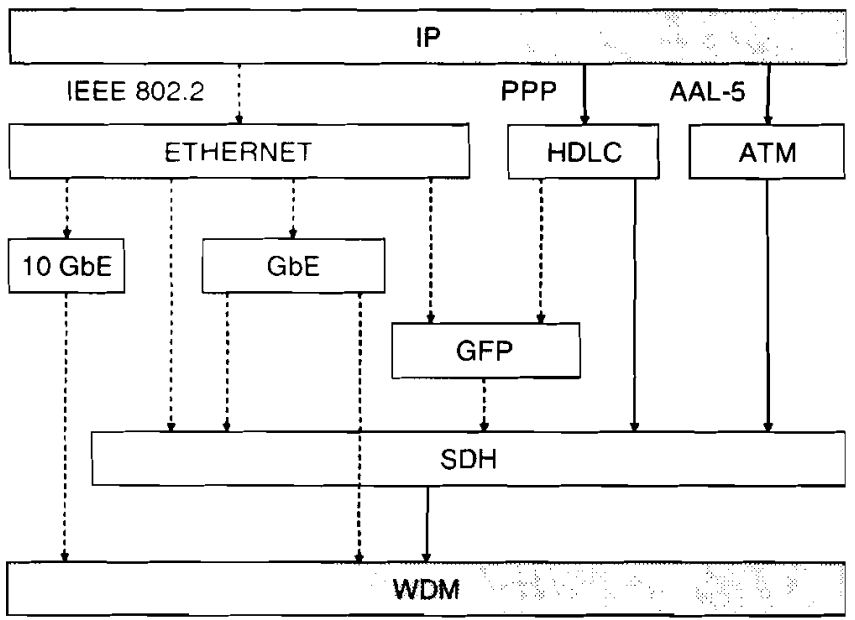

Figura 2. Algumas técnicas de encapsulação de tráfego IP para transporte em redes ópticas WDM. As setas contínuas indicam as arquiteturas citadas neste artigo. AAL-5: ATM adaptation laver type 5; GFP: generic framing procedure; PPP: point-to-point protocol; GbE: gibabit Ethernet; HDLC: high-level data link control.

O desenvolvimento do padrão GbE está a cargo do grupo de trabalho IEEE 802.3 que criou, em 1996, o 802.3 Gigabit Ethernet Task Force [25]. Os dois padrões em desenvolvimento são o Gigabit Ethernet (GbE) e o 10 Gigabit Ethernet (10GbE) [26], que utilizam o formato de quadros de tamanho variável. Estes protocolos podem se utilizados em redes locais de alcance até $40 \mathrm{~km}$ sobre fibra (10GbE). No caso do padrão GbE, a encapsulação de pacotes IP sobre este padrão acrescenta ao cabeçalho uma quantidade significativa de bytes [27].

Outras duas técnicas de encapsulação são a IP sobre SONET/SDH, utilizando o protocolo IP/PPP/HDLC (IP/point-to-point protocol/high-level data link control) [28], e a IP sobre ATM [29].

Uma forma de comparar o desempenho das várias arquiteturas multicamadas é estimar a penalidade associada à quantidade de bytes que compõem o cabeçalho.

As duas últimas técnicas mencionadas (IP/PPP/HDLC e IP sobre ATM) foram escolhidas neste artigo para uma comparação direta, para as quais o acréscimo de bytes (penalidade de cabeçalho) foi calculado por intermédio da análise de cinco tamanhos (bytes) de pacotes IP, correspondente a uma amostra de cinco minutos (mais de 18 milhões de pacotes, totalizando 6,7 Gbytes) de um enlace STM-1 (synchronous transport module, 155,52 Mbps) do backbone da MCI Telecommunications Corporation [30]. Os resultados são apresentados na Tabela 1 , sem considerar o cabeçalho inserido pela infra-estrutura de transporte SONET/SDH (aproximadamente $4,6 \%$ ). Notase que $38,9 \%$ e $10,1 \%$ do total de pacotes do backbone são compostos de 40 e 44 bytes, respectivamente.

$O$ cabeçalho inserido pela encapsulação IP/ATM, para cada pacote de informação, é formado pelo cabeçalho de célula ( 5 bytes) mais 11 bytes inseridos pela encapsulação AAL-5 (ATM Adaptation Layer Type 5), totalizando 16 bytes. Tanto pacotes de 40 quanto de 44 bytes não podem ser encapsulados em uma única célula ATM por meio da encapsulação IP/ATM.

Cada célula ATM é formada de 48 bytes reservados para a informação (payload) mais 5 bytes de cabeçalho. Assim, um pacote IP com 40 bytes seria acrescido de 16 bytes, totalizando 56 bytes, que seriam distribuídos entre duas células ATM. Uma das células transportaria 48 bytes mais o respectivo cabeçalho de 5 bytes, enquanto a outra transportaria 8 bytes de informação e 40 bytes de enchimento (totalizando 48 bytes) mais o cabeçalho de 5 bytes. A quantidade resultante seria 66 bytes $(16+40+2 \times 5)$ e a penalidade em relação aos 40 bytes do pacote IP original, $165 \%$. No caso de pacotes IP de 44 bytes, a penalidade seria $140,9 \%$. Portanto, a encapsulação IP/ATM se mostra extremamente ineficiente para estes dois tamanhos de pacotes. Para pacotes maiores, a distribuição dos bytes entre várias células ATM é mais equilibrada e o acréscimo é menor.

Por outro lado, a encapsulação IP sobre SONET/SDH acrescenta 8 bytes, independentemente do tamanho do pacote IP a ser mapeado.

Consideremos agora o acréscimo ponderado médio de bytes, definido pela relação $x / y$, na qual $x=\sum_{i}^{5}\left(b_{I P / A T M} \times t p_{I P}\right) / t p_{I P}, \quad y=\sum_{I}^{5}\left(b_{l p} \times t p_{I P}\right) / t p_{I P}, b_{I P / A T M}$ é o número de bytes utilizados pela encapsulação IP sobre WDM, $b_{I P}$ é o número de bytes do pacote IP e $t p_{I P}$ é o total de pacotes IP. No caso dos cinco tamanhos de pacotes IP 
listados na Tabela 1, o acréscimo médio ponderado de bytes devido à encapsulação IP sobre ATM é de aproximadamente $24 \%$, enquanto para a encapsulação de IP/PPP/HDLC é de, aproximadamente, 2\% $(x=8)$. Portanto, a abordagem IP sobre DWDM demonstra superioridade em relação às outras possibilidades. Para tal, a tecnologia MPLS provida de capacidade de engenharia de tráfego, MPLS-TE (traffic engineering), proporcionará a mesma capacidade de alocação de canais virtuais típica do ATM. Esta funcionalidade poderá ser empregada na substituição da camada ATM. O conceito básico desta tecnologia será o tema da próxima seção.

\begin{tabular}{|c|c|c|c|c|}
\hline \multicolumn{2}{|c|}{$\begin{array}{c}\text { Pacotes IP no } \\
\text { backbone da MCI } \\
\text { Telecom. [30] }\end{array}$} & \multicolumn{3}{|c|}{ Penalidade de cabeçalho } \\
\hline $\begin{array}{c}\text { Tamanho } \\
\text { dos } \\
\text { pacotes } \\
\text { (bytes) }\end{array}$ & $\begin{array}{c}\text { Total de } \\
\text { pacotes } \\
(\%)\end{array}$ & \multicolumn{2}{|c|}{ IP sobre ATM } & $\begin{array}{c}\text { IP sobre } \\
\text { SONET }\end{array}$ \\
\cline { 3 - 5 } & $\begin{array}{c}\text { Bytes } \\
\text { utilizados } \\
\text { (total) }\end{array}$ & $(\%)$ & $(\%)$ \\
\hline 40 & 38,9 & 66 & 165,0 & 20,0 \\
\hline 1500 & 11,5 & 196 & 13,1 & 0,5 \\
\hline 552 & 10,1 & 84 & 15,2 & 1,4 \\
\hline 44 & 6,1 & 62 & 140,9 & 18,2 \\
\hline 576 & 4,9 & 113 & 19,6 & 1,4 \\
\hline
\end{tabular}

Tabela 1. Comparação entre as penalidades de cabeçalhos inseridos pela encapsulação IP sobre SONET e IP sobre ATM para cinco tamanhos de pacotes IP [30]. IP: internet protocol; SONET: synchronous optical network; ATM: asynchronous transfer mode.

\section{ARQUITETURA MPLS E IMPLEMENTAÇÃO DE ENGENHARIA DE TRÁFEGO EM REDES IP}

O uso de arquitetura MPLS objetiva viabilizar a implementação de engenharia de tráfego em redes IP. A engenharia de tráfego trata da alocação eficiente de fluxos de tráfego IP nos recursos físicos existentes em uma rede, enquanto atende simultaneamente às exigências de qualidade desejada (atrasos e níveis de proteção, por exemplo) para o transporte das informações contidas nos pacotes. Há diversas técnicas para atingir este objetivo e uma delas é a arquitetura MPLS. O termo multiprotocol significa que esta tecnologia pode ser usada com qualquer protocolo de rede (IP e IPX, por exemplo).

$\mathrm{O}$ fundamento consiste em designar rótulos aos pacotes na borda de uma rede MPLS. No interior do domínio MPLS os rótulos anexados aos pacotes são usados para a tomada de decisões de envio, sem a necessidade de analisar os cabeçalhos originais dos referidos pacotes. Desta maneira, a transmissão de dados ocorre nos LSPs (label switched path), os quais são uma seqüência de rótulos designados em cada nó ao longo do caminho fonte-destino.

Os rótulos são distribuídos por intermédio de protocolos como o CR-LDP (constraint based routing with label distribution protocol), RSVP ou, ainda, via protocolos de roteamento como o BGP (border gateway protocol). A escolha recai sobre aquele protocolo que melhor atender às exigências de operação da rede, não sendo imposta pela arquitetura MPLS. Cada pacote encapsula e carrega o rótulo durante o trajeto fonte-destino. $O$ processamento dos pacotes é realizado rapidamente porque não há necessidade de consultar o cabeçalho do pacote para comutá-lo entre enlaces.

Os LSPs podem ser configurados de maneira explícita antes de ocorrer a transmissão de dados, ou seja, os nós pelos quais os pacotes trafegarão podem ser especificados antecipadamente. Ao longo do caminho, os recursos podem ser reservados para assegurar QoS para este tráfego de dados. Tal característica efetua engenharia de tráfego ao longo da rede, pois permite a utilização de outros caminhos que não necessariamente os mais curtos (estes usualmente selecionados pelos protocolos convencionais de roteamento IP), resultando em otimização de recursos. Por exemplo, pode-se mover o fluxo de dados para uma nova rota se o caminho mais curto entre dois nós estiver congestionado. Alternativamente, as características desejadas para um LSP, como largura de faixa, destino ou prioridade, podem ser configuradas em um roteador que usa um algoritmo apropriado para selecionar o caminho através da rede que satisfaça as restrições impostas.

O MPLS torna-se, desta maneira, um instrumento apropriado para estabelecer e manter caminhos explicitamente roteados em uma rede IP para uma efetiva alocação de tráfego.

Embora o MPLS ofereça um método para a configuração de caminhos e envio de tráfego, uma questão ainda não abordada é como encontrar caminhos com restrições. Para habilitar a determinação de rotas com restrições, o IETF tem como objetivo estender os IGPs (interior gateway protocol) já utilizados, como o OSPF [31] e o IS-IS [32], para transportar informações adicionais sobre os enlaces. Tais informações incluem largura de faixa máxima, bem como prioridade para realização de reserva de recursos, baseada em oito níveis de prioridades e métricas relacionadas à engenharia de tráfego. Métricas como estas tornam os processos de roteamento livres das restrições da métrica usada pelos IGPs, geralmente relacionadas ao caminho mais curto. A informação do estado da topologia pode então ser usada para selecionar possíveis rotas para os LSPs.

De maneira geral, o IETF tem procurando estender não só os protocolos IGPs para trabalhar com restrições de rotas em redes MPLS, como também a própria topologia MPLS para trabalhar como plano de controle em redes ópticas. Este tema será abordado na próxima seção.

\section{ARQUITETURA GMPLS}

A função da arquitetura GMPLS [33] é prover as redes ópticas de um plano de controle comum para configurar conexões dinamicamente. O objetivo é oferecer um LSP ótimo baseado nos requisitos de tráfego de um usuário. Tal LSP pode, por exemplo, começar em uma rede IP, ser transportado por uma rede SONET/SDH e ser comutado por meio de um determinado comprimento de onda em uma fibra óptica. Um diagrama de blocos do plano de controle GMPLS pode ser visto na Figura 3. 


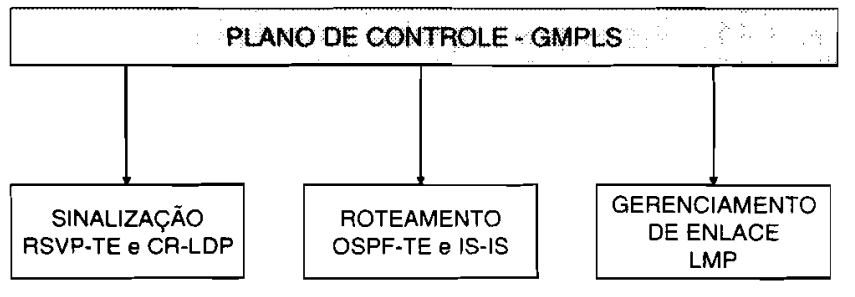

Figura 3. Diagrama de blocos da arquitetura GMPLS. RSVP-TE: resource reservation protocol-traffic engineering; CR-LDP: constraint-based routing-label distribution protocol; OSPF: open shortest path first; IS-IS: interior system to interior system; LMP: link management protocol.

Estes blocos são baseados em protocolos de sinalização e roteamento já conhecidos, com extensões para suportar o GMPLS. Somente um novo protocolo é exigido, o de sinalização para gerenciamento de enlace (LMP-link management protocol).

De fato, o uso de tecnologias como o WDM possibilita a existência de um grande número de enlaces paralelos entre dois nós adjacentes. Com isto, a configuração manual e o controle destes enlaces tornam-se impraticáveis. Por isso, o LMP foi especificado para resolver estas questões, provendo mecanismos para:

a) Manter a conectividade do canal de controle. A arquitetura GMPLS permite que o plano de dados seja separado fisicamente do plano de controle, possibilitando a criação de canais de controle, pelos quais são transmitidas as mensagens de sinalização e roteamento;

b) Verificar a conectividade física dos enlaces (link verification):

c) Realizar processo de correlação para determinação de inconsistências e propriedades dos enlaces (link property correlation);

d) Gerenciar as falhas dos enlaces (fault link and fault notification).

Além disso, extensões para protocolos de roteamento tradicionais, como o OSPF [34] e o IS-IS [35], são necessárias para codificar e transportar uniformemente informações de enlace, como o estado e as restrições ao uso de certos recursos físicos e suas propriedades (identificadores de fibras ópticas, largura de faixa e comprimentos de onda) entre dois nós GMPLS. Adicionalmente, a sinalização [36] deve agora ser capaz de transportar os parâmetros requeridos pelo LSP, como largura de faixa, tipo de sinal, proteção desejada e posição em um dispositivo TDM, além de fibras ópticas. Assim, o GMPLS é capaz de estender as funções dos dois protocolos de sinalização, RSVP-TE [37] e CR-LDP [38].

Até o momento, a padronização da arquitetura GMPLS possui oito RFCs (request for comment), designadas por RFC 3471, 3472, 3473, 3474, 3609, 3945, 3946 e 4003 [10].

\subsection{ESTABELECIMENTO DE LSP EM REDES GMPLS}

O estabelecimento de um LSP em redes GMPLS é um processo similar ao realizado em redes MPLS. A Figura 4 mostra a configuração de um LSP através de múltiplos enlaces de diferentes tecnologias [18]. A seguir são descritas a composição dos enlaces e a configuração de um LSP.

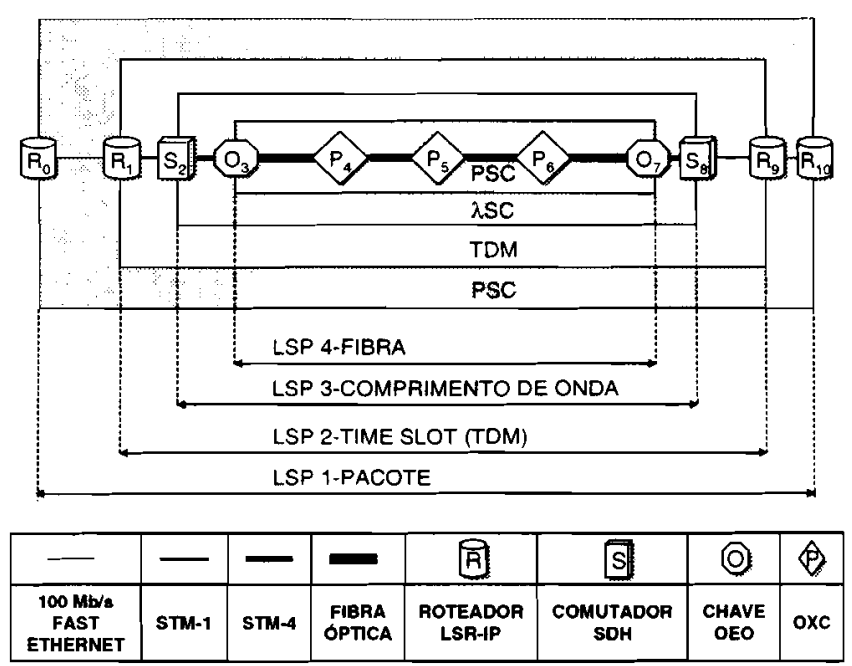

Figura 4. Configuração de um LSP através de múltiplos enlaces de diferentes tecnologias. O enlace entre $R_{0}$ e $R_{l}$ é Fast-Ethernet; entre $R_{1}$ e $S_{2}$ é STM-1: entre $S_{2}$ e $O_{3}$ é STM4, especificado em um comprimento de onda; entre $O_{3}$ e $O_{7}$ é um sistema WDM com 16 comprimentos de onda, cada um capaz de transportar um STM-4. O enlace entre $O$, e $S_{s}$ é um STM-4; entre $S_{8}$ e $R_{9}$ é STM-1; entre $R_{9}$ e $R_{b 0}$ é FastEthernet. PSC: packet switch capable; LSC: lambda switch capable; STM: synchronous transport module; TDM: time division multiplexing.

\subsubsection{COMPOSIÇÃO E FUNCIONALIDADE}

De acordo com o esquema mostrado na Figura 4, o roteador $R_{0}$ envia pacotes para o roteador $R_{l}$ que dispõe de interface STM-l e os aloca em janelas temporais (time slots). No outro extremo, o roteador $R_{9}$ extrai os pacotes das janelas temporais e os entrega ao roteador $R_{10} . S_{2}$ e $S_{\delta}$ são comutadores SDH. Os dispositivos $O_{3}$ e $O_{7}$ são comutadores ópticos, cuja função é comutar o enlace STM-4 recebido sob a forma de um comprimento de onda para um sistema WDM. Este comprimento de onda será comutado pelos OXCs $P_{4}$ a $P_{6}$. O enlace entre $R_{0}$ e $R_{l}$ é Fast-Ethernet; entre $R_{I}$ e $S_{2}$ é STM-1; entre $S_{2}$ e $O_{3}$ é STM-4, especificado em um comprimento de onda; entre $O_{3}$ e $O_{7}$ é um sistema WDM com 16 comprimentos de onda, cada um capaz de transportar um STM-4. Tais comprimentos de onda são comutados pelos OXCs. O enlace entre $O_{7}$ e $S_{8}$ é um STM-4 recebido em forma de um comprimento de onda; entre $S_{8}$ e $R_{9}$ é STM-1; entre $R_{9}$ e $R_{l o}$ é Fast-Ethernet.

\subsubsection{CONFIGURAÇÃO DE UM LSP}

A configuração de um LSP é realizada da seguinte forma (Figura 4). O LSP1 é configurado de R0 até R10 com 
largura de faixa de $100 \mathrm{Mbps}$. O LSP1 é agrupado nos LSPs 2, 3 e 4 , respectivamente. Neste exemplo, a largura de faixa requerida pelo LSPI é de $100 \mathrm{Mbps}$, devendo a rota ter suficiente largura de faixa em toda sua extensão para satisfazer tal exigência. O roteador R0 mapeia os pacotes para o LSPI. Esice será agrupado pelo LSR R1 no LSP2 com taxa de 155 Mhps (STM-1). O comutador (switch) S2 multiplexa o sinal de 155 Mbps e o torna disponível ao LSP3. que dispoc de 622 Mbps (STM-4 entre S2 e O3), escolhe um comprimento de onda e o envia ao comutador óptico 03 . Estc comutador óptico recebe o comprimento de onda selecionalo em uma de suas portas e o comuta através do LSPt pilra $\mathrm{lm}$ canal WDM até P4. Este é comutado através de P5 ¿'P6 até O7. Deste modo, O7 seleciona o comprimento de onda correto e o transmite à porta

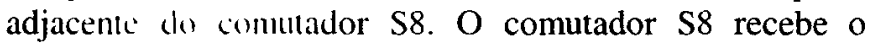
referido comprimento de onda, demultiplexa o sinal, selecionando "STM-1 desejado dentre aqueles que constituem o nicl hierárquico STM-4, e o transmite para o LSR R9. Finalmente, o LSR R9 recebe os pacotes do STMI e os enria para o LSR R10.

O processu de sinalização para o LSP1 é descrito na Figura 5 uilisando, como exemplo, o protocolo de sinalizaçīo RSIP ' suas extensões definidas na arquitetura GMPLS ||$S \mid$.

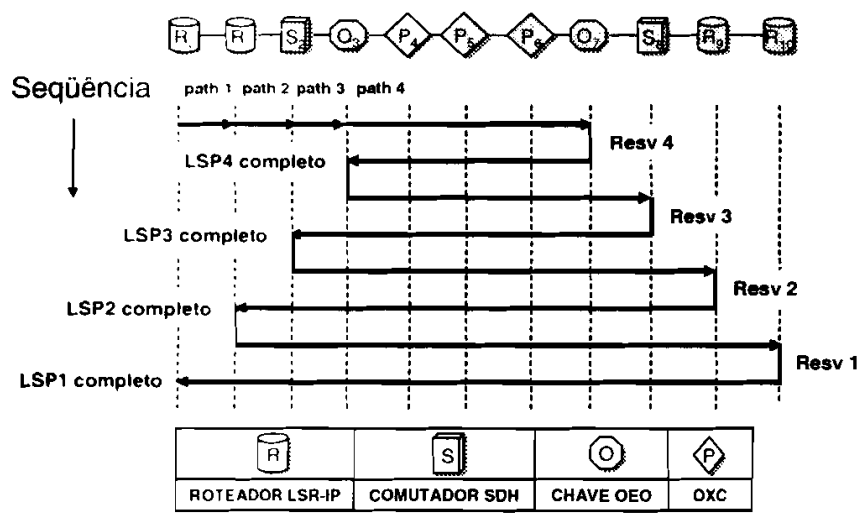

Figura 5. Unu mensagem path request (path 1) é gerada no roteador $R($ ) ‘ enviada a $R I$. No roteador $R 1$ este pedido dá origem a uma solicitação para um novo LSP (LSP2) de R1 a R9. Esta dinâmica de criação é repetida até que a mensagem path + seja gerada no comutador O3. Em seguida à configuração do LSP4, traduzido pelo recebimento da mensagem Resv, o LSP3 é "tunelado" através do LSPt. Este processo de formação de LSP, no qual un pedido de LSP de baixa ordem é canalizado através de um LSP já estabelecido de ordem mais alta, continua até que o LSP inicial (LSPI) seja configurado.

Geralmente, um LSP é estabelecido pelo envio de mensagens path (RSVP)/label request (CR-LDP) para o nó de destino (nó donnstream). Estas mensagens contêm um pedido de rótulo genérico (generalized label request) com os tipos de LSP (isto é, a camada envolvida) e de carga, bem como parâmetros específicos, como informação de proteção (protection information). Adicionalmente, uma sugestão de rótulo (label suggestion), um conjunto de rótulos (label set), um upstream label (se o LSP é bidirecional) e a informação do status administrativo do LSP tambén podem ser incluídos nesta mensagem. Uma rota explicita é normalmente adicionada à mensagem pelo primeiro LSR: O nó de destino retornará uma mensagem Resv (RSVP)/label mapping (CR-LDP), incluindo um rótulo genérico (generalized label) com $\mathrm{O}$ intuito de configurar o LSP.

A inserção do GMPLS na arquitetura de redes ópticas padronizada pelo ITU-T justifica-se, uma vez que elas utilizam um plano de controle que pode ser composto pelos protocolos empregados pelo plano de controle GMPLS. Este tema será abordado na próxima seção.

\subsection{MODELOS DE INTERCONEXÃO DE CAMADAS}

A arquitetura GMPLS permite a implementação de dois tipos de interconexão entre as camadas: o modelo overlay e o modelo peer [18]. Estes modelos foram propostos a partir de diferentes procedimentos para a solicitação de serviços à rede e podem ser vistos como balizadores na evolução da padronização das redes ópticas.

O modelo peer torna o núcleo de uma rede óptica acessível, permitindo aos dispositivos de borda (cliente) ter acesso à topologia da rede e participar das decisões de roteamento, eliminando a barreira artificial entre o domínio de transporte e roteamento. Este acesso à topologia da rede permite que os mesmos protocolos de sinalização empregados na arquitetura GMPLS sejam utilizados para configurar um LSP através da rede.

O modelo overlay não permite aos dispositivos de borda acesso à topologia da rede e o núcleo e os dispositivos de borda são gerenciados separadamente a partir de diferentes protocolos de sinalização. Por este motivo, o modelo overlay tem estimulado a parceria entre o OIF e o IETF para fins de padronização.

Como resultado desta parceria, foi desenvolvida uma interface denominada UNI (user to network interfaceOIF), que permite aos dispositivos clientes da rede a solicitação dinâmica de conexões. Uma característica fundamental da UNI é não permitir aos clientes o acesso às informações da topologia ou endereçamento da rede. Uma solicitação de cliente deve identificar os pontos extremos de uma conexão, a fonte (ele próprio) e o destino, usando algum esquema de endereçamento. De acordo com esta estratégia, é designado um endereço TNA (transport network assigned address) a cada nó cliente de uma rede. Desta maneira, os clientes utilizam-se de TNAs, enquanto a encapsulação entre os endereços designados e os endereços internos de uma rede é feito dentro das fronteiras da rede.

Embora a UNI não seja parte integrante do padrão GMPLS, a função de sinalização requerida está muito próxima daquela oferecida pelos protocolos de sinalização RSVP e CR-LDP. Com algumas extensões para suportar características da UNI, como endereçamento TNA e identificação de conexão, estes protocolos de sinalização podem ser utilizados para a sinalização UNI. Similarmente, o gerenciamento de vários enlaces UNI pode ser alcançado por extensões do protocolo LMP.

O OIF produziu um documento de especificação denominado UNI-OIF, que descreve como os protocolos existentes podem ser estendidos para proporcionar as funcionalidades da interface [9]. 


\section{PADRONIZAÇÃO DE REDES ÓPTICAS NO ÂMBITO DO ITU-T E A INSERÇÃO DA ARQUITETURA GMPLS}

O conceito ASON foi proposto, no âmbito do ITU-T, com intuito de permitir o transporte em redes WDM de vários padrões, como SONET/SDH, Ethernet e ATM, operando sob gerenciamento unificado. Com este objetivo, - ITU-T elaborou uma lista de recomendações que especificam o plano de controle, o plano de transporte, o plano de gerenciamento e as DCNs (data comunications networks). As DCNs são utilizadas para formar uma rede de comunicação, na qual são trocadas as informações de gerenciamento e/ou sinalização entre os diversos planos. As recomendações pertinentes estão reunidas no Apêndice, Tabelas 2-5.

As recomendações são implementadas a partir de uma abordagem por fases, dependendo da maturidade da tecnologia envolvida e das necessidades do mercado. $\mathrm{Na}$ fase 1, na qual está a maioria delas, a ênfase é dada aos sistemas ponto a ponto e, na fase 2 , à criação de extensões para a implantação de OADMs e OXCs. As recomendações ITU-T G.871, G.872 e G.959.1 não estão sujeitas à abordagem por fases.

De forma geral, o plano de transporte estabelece uma rota (pacotes, janelas temporais ou comprimento de onda) na qual trafegam os dados do usuário. $O$ plano de gerenciamento monitora e administra o plano de transporte. O plano de controle provê a configuração e extinção de circuitos (caminhos) por meio de sinalizações distribuídas, habilitando novos serviços de rede como, por exemplo, largura de faixa por encomenda (BoD-bandwidth on demand). Assim, o plano de controle em uma rede ASON tem a função de estabelecer conexões por meio de sinalização automática, evitando a operação manual da rede. As recomendações que especificam o plano de controle no âmbito do ITU-T são apresentadas na Figura 6.

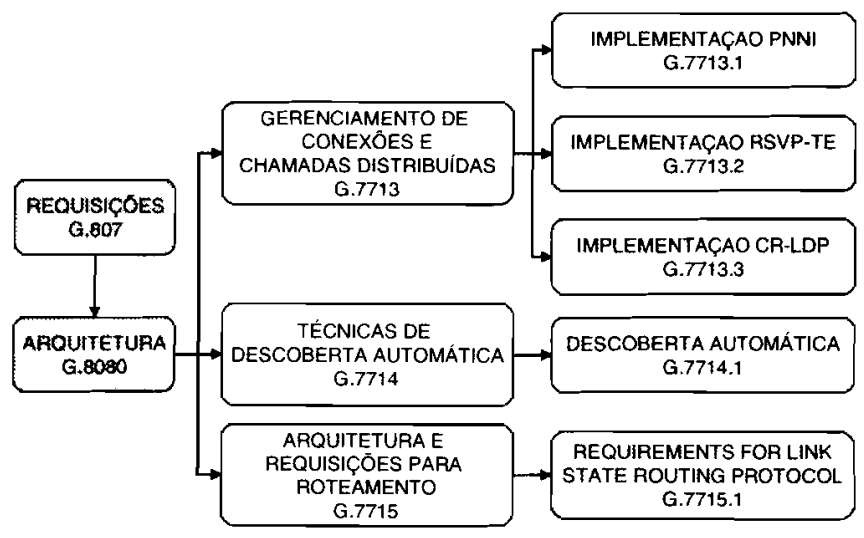

Figura 6. Recomendações que descrevem o plano de controle ASON. PNNI: private network-private interface; RSVP-TE: resource reservation protocol-traffic engineering; CR-LDP: constraint-based routing- label distribution protocol.

A fim de prover as redes ópticas de mecanismos para o estabelecimento de chamadas e conexões de maneira automática, é necessário conhecer os recursos disponíveis.
Estes recursos podem ser notificados manualmente ou descobertos automaticamente. A descoberta automática da topologia e dos recursos é o assunto das recomendações ITU-T G.7714 e ITU-T G.7714.1. Esta recomendação não implementa nenhum protocolo de descoberta automática de recursos, mas apenas caracteriza, de maneira geral, a troca de mensagens para determinar a existência de conectividade entre dois elementos. Por exemplo, a mensagem "HELLO" deverá ser utilizada nos enlaces para a detecção de conectividade. A recomendação ITU-T G.7715 especifica os requisitos e a arquitetura das funções de roteamento utilizadas para a configuração de conexões em uma rede ASON. A recomendação ITU-T G.7715.1 especifica os requerimentos necessários aos protocolos de roteamento por estado de enlace, sendo neutra em relação a tais protocolos.

A arquitetura de roteamento estabelece uma segregação hierárquica, ou seja, a topologia de uma rede ASON é descrita por domínios, visando tornar tais redes expansíveis. Tais domínios podem ser divididos em áreas, tornando mais racional o processo de roteamento. Em outras palavras, dentro destas áreas de roteamento são trocadas informações locais detalhadas e informaçōes menos detalhadas sobre áreas remotas. Áreas de roteamento podem ser divididas recursivamente, criando uma hierarquia de informações. A recomendação ITU-T G.7715 estabelece os blocos funcionais que serão utilizados para as funções de roteamento em redes $\mathrm{ASON}$, não especificando qualquer tipo de protocolo.

A arquitetura do plano de controle é apresentada na recomendação ITU-T G.8080. A recomendação ITU-T G.7713 cobre aspectos associados ao plano de controle ASON, estabelecendo os requisitos de sinalização necessários para a comunicação dos componentes do plano. As recomendações ITU-T G.7713.1, G.7713.2, G.7713.3 implementam diferentes protocolos para a execução das funções de sinalização.

\section{PROTOCOLOS GMPLS E PONTOS DE REFERÊNCIA ASON}

O plano de controle ASON supõe que a rede é formada por domínios que interagem com outros domínios de maneira padronizada. Tais domínios podem ser divididos, por exemplo, em domínios geográficos ou domínios utilizando o mesmo tipo de equipamento. As interfaces entre domínios, como ilustra a Figura 7, são conhecidas como pontos de referência, sendo denominados: UNI (user network interface), E-NNI (exterior node to node interface) e I-NNI (interior node to node interface). Estes pontos de referências indicam a localização na qual mensagens de sinalização e roteamento são trocadas utilizando protocolos padronizados.

De fato, o plano de controle GMPLS se refere a um conjunto de protocolos que, quando for completamente especificado, poderá habilitar operação fim a fim entre os dispositivos das redes ópticas. Os protocolos são os seguintes:

a) RSVP-TE generalizado, para sinalização;

b) CR-LDP generalizado, para sinalização;

c) OSPF com extensões TE (traffic engineering) para roteamento intra-área; 
d) IS-IS (interior system to interior system) com extensões TE para roteamento intra-área;

e) LMP para realizar o gerenciamento de enlaces e implementar funções de descobertas.

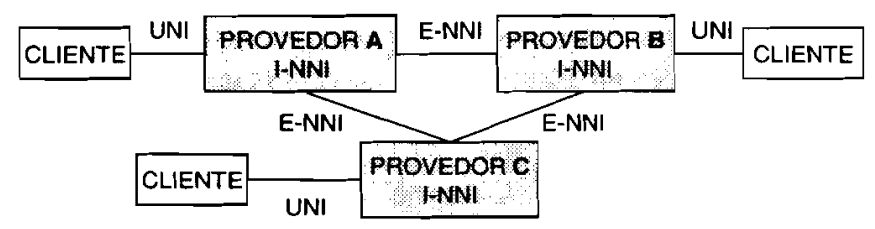

Figura 7. Rede ASON (automatically switched optical networks) e a divisão de domínios e os pontos de referências: UNI (user network interface); I-NNI (interior node to node interface); E-NNI (exterior node to node interface).

Os protocolos RSVP-TE e CR-LDP são protocolos de sinalização que efetivamente desempenham funções idênticas. Foram implementados por causa de conflitos de interesses entre seus propositores, presentes entre os membros da IETF. Os protocolos OSPF-TE e IS-IS são também equivalentes. Protocolos de roteamento interáreas para o ambiente óptico não foram definidos até esta data.

Quanto à arquitetura do plano de controle ASON (Figura 3), o ITU-T elaborou recomendações que incorporam protocolos presentes na arquitetura GMPLS (RSVP-TE e CR-LDP), podendo ser constatada a existência de vários protocolos de sinalização para executar a mesma função. Tais protocolos serão empregados nos pontos de referência UNI, E-NNI e I-NNI. O ponto de referência UNI permite aos clientes ASON criar conexões, isto é, eles podem sinalizar para a rede com o intuito de estabelecer novas conexões com atributos específicos. Tais atributos podem incluir largura de faixa, proteção e restauração. Ademais, permitem interromper ou modificar uma conexão.

A respeito da especificação da interface UNI, cuja sinalização é voltada para solicitar serviços que a rede de transporte oferece aos clientes, o OIF aprovou em 2004 a especificação User Network interface $1.0-$ signaling specification —OIF-UNI-1.0-Release 2 [9]. Esta especificação descreve as regras gerais para a implantação de serviços, como o estabelecimento e interrupção de conexão, mudança de status e tráfego. Além dos serviços, ela define os protocolos de sinalização, o mecanismo de transporte de mensagens de sinalização e os procedimentos para autodescoberta.

Com objetivo de estabelecer regras específicas para diferentes protocolos de sinalização, o OIF apresentou, neste mesmo ano, a especificação RSVP Extensions for User Network Interface (UNI)-1.0 Signaling e a especificação Intra-Carrier E-NNI Signaling Specification, que descrevem extensões para os protocolos RSVP-TE, CRLDP e PNNI para habilitar configurações dinâmicas no ponto de referência E-NNI [9].

Formas de implementar extensões para incluir suporte a chamadas, no âmbito do IETF, para os protocolos RSVPTE e CR-LDP, podem ser encontradas em [39] e [40], respectivamente. Para o ponto de referência I-NNI, os protocolos de roteamento OSPF ou IS-IS podem ser utilizados segundo o IETF. Já para o ponto de referência ENNI, o IETF não tem uma posição definida e está considerando algumas opçóes como, por exemplo, criar extensões para o protocolo BGP ou estender protocolos como OSPF ou IS-IS para operar roteamento entre domínios. Por outro lado, o ITU-T não definiu quais protocolos de roteamento utilizará nos referidos pontos de referência.

As recomendações do ITU-T que especificam a rede de transporte óptica (ASON) estão reunidas em tabelas no Apêndice.

\section{CONCLUSÕES}

A evolução das redes ópticas será ditada pela nova base tecnológica representada por amplificadores ópticos de faixa cada vez mais larga, novas fibras ópticas e OADMs e OXCs totalmente ópticos. A viabilidade tecnológica destes dispositivos possibilitará a redução do número de camadas existente entre a camada de aplicação e a camada física visando melhor atender a demanda por serviços.

Neste artigo, a evolução das redes ópticas foi avaliada e as limitações de desempenho devido à inserção de cabeçalhos das pilhas de protocolos IP/ATM/SONET-SDH e IP/HDLC/SONET-SDH foram analisadas, demonstrando a necessidade de as redes evoluírem para uma topologia mais eficiente. Os fundamentos dos protocolos MPLS e GMPLS foram descritos, assim como a estrutura do plano de controle GMPLS. A inserção da arquitetura GMPLS à arquitetura ASON, padronizada pelo ITU-T, foi avaliada. Esta inserção é justificada porque as redes ópticas padronizadas pelo ITU-T utilizam um plano de controle que pode ser implementado pelos protocolos empregados pelo plano de controle GMPLS. As especificações propostas pelos organismos ITU-T, OIF e IETF foram sistematicamente reunidas e discutidas, considerando o seu estágio atual de desenvolvimento.

\section{AGRADECIMENTOS}

Os autores agradecem o apoio da Fundação Centro de Pesquisa e Desenvolvimento em Telecomunicações (CPqD) e da Financiadora de Estudos e Projetos (FINEP), com recursos financeiros do Fundo para o Desenvolvimento Tecnológico das Telecomunicações (FUNTTEL), via Projeto Giga. Agradecem também o apoio da FAPESP, via Projeto Tidia-KyaTera (proc. no. 2003/08269-7).

\section{REFERÊNCIAS}

[1] J.P. Ryan e R.H. Kent. "WDM: North American Deployment Trends", IEEE Communications Magazine, v.36, n.2, pp. 4044, fevereiro de 1998.

[2] C.A. Siller e M. Shafi (editores), SONET/SDH: A Sourcebook of Synchronous Networking, Piscataway, NJ, EUA: IEEE Press, 1996.

[3] M. Sexton e A. Reid, Broadband Networking: ATM, SDH, and SONET. Norwood, MA, EUA: Artech House, 1997.

[4] Y. Tze-Wei, K.L. Eddie e A. Goldenberg, "MEMS Optical Switches", IEEE Communications Magazine, v.39, n.1 1, pp.1 58-163, novembro de 2001.

[5] Multiprotocol Label Switching (MPLS). Disponível em www.iec.org/online/tutorials/acrobat/mpls.pdf. 
[6] E. Rosen, A. Viswanathan e R. Callon, "Multiprotocol Label Switching Arquiteture", IETF RFC 3031, janeiro de 2001. www.ietf.org.

[7] Generalized Multiprotocol Label Switching (GMPLS). Disponível www.iec.org/online/tutorials/gmpls/topic04.html.

[8] International Telecommunication Union (ITU). Disponível em www.itu.int.

[9] Optical Internetworking Forum (OIF). Disponivel em www.oiforum.com.

[10] The Internet Engineering Task Force (IETF). Disponivel em www.ietf.org.

[11] Fundação Centro de Pesquisa e Desenvolvimento em Telecomunicações $\quad(\mathrm{CPqD}) . \quad$ Disponivel em www.cpqd.com.br

[12] Rede Nacional de Ensino e Pesquisa (RNP). Disponível em www.rnp.br.

[13] Fundo para o Desenvolvimento Tecnológico das Telecomunicações (FUNTTEL). Disponível em www.mc.gov.br/funttel.

[14] Financiadora de Estudos e Projetos (FINEP). Disponível em www.finep.gov.br.

[15] Projeto GIGA. Disponível em www.projelogiga.org.br.

[16] Programa Tecnologia de Informação no Desenvolvimento da Internet Avançada (TIDIA). Fapesp. Disponível em http://tidia-ae.incubadora.fapesp.br/home.

[17] Projeto Kyatera-Tidia-Fapesp. Disponível em www.kyatera.fapesp.br/.

[18] A. Banerjee et al., "Generalized Multiprotocol Label Switching: An Overview of Routing and Management Enhancements", IEEE Communications Magazine, pp. 144150 , janeiro de 2001.

[19] A. Banerjee et al., "Generalized Multiprotocol Label Switching: An Overview of Signaling and Enhancements and Recovery Techniques", IEEE Communications Maga-ine, pp. 144-151, julho de 2001.

[20] S. Tomic, B.S.-Halimi e A. Halimi, "ASON and GMPLSOverview and Comparison", Photonic Network Communications, vol. 7. no. 2, pp. 111-130, 2004.

[21] Eduardo José Aloia e Murilo Araújo Romero (orient.), "Sistematização Crítica das Tendências de Padronização de Arquiteturas e Protocolos em Redes Ópticas". Dissertação de mestrado. EESC-USP, 2003, 159 p.

[22] Recomendação ITU-T G 694.1, "Spectral grids for WDM applications: DWDM frequency grid", 2002. Disponível em www.itu.int.

[23] A. Borella, G. Cancellieri e F. Chiaraluce, Wavelength Division Multiple Acess Optical Networks. Norwood, MA, EUA: Artech House, 1998.

[24] ATM Forum. Disponível em www.atmforum.com.

[25] IEEE 802 LAN/MAN Standards Committee. Disponivel em http://www.ieee802.org/

[26] IEEE P802.3ae 10Gb/s Ethernet Task Force. Disponível em http://grouper.ieee.org/groups/802/3/ae/

[27] H.-M. Foisel, M. Jaeger, F.-J. Westphal, K. Ovsthus e J.-C. Bischoff, "Evaluation of IP over WDM Network Architetures", Photonic Network Communications, 3:1/2, pp. 41-48, 2001.

[28] Malis e W. Simpson, "PPP over SONET/SDH", IETF RFC 2615, junho de 1999.

[29] M. Laubach. "Classical IP and ARP over ATM", IETF RFC 1577, janeiro de 1994. Disponível em www.ietf.org.

[30] J. Anderson et al, "Protocols and architectures for IP Optical Networking", Bell Labs Technical Joumal, v.4, n.1, p.105124, janeiro-março de 1999.

[31] D. Katz, D. Yeung e K. Kompella, "Traffic Engineering Extensions to OSPF Version 2", IETF, RFC 3630, setembro de 2003. Disponível em www.ietf.org.
[32] H. Smit e T. Li, "IS-IS Extensions for Traffic Engineering". IETF, RFC 3784, junho de 2004. Disponivel em www.ietf.org.

[33] E. Mannie et al, "Generalized Multi-Protocol Label Switching (GMPLS) Architecture". IETF, RFC 3945. outubro de 2004. Disponível em www.ietf.org.

[34] K. Kompella et al, "OSPF Extensions in Support of Generalized MPLS". IETF, internet draft, RFC Ed Queue. Em fase de desenvolvimento, outubro de 2003. Disponível em www.ietf.org/internet-drafts/draft-ietf-ccamp-ospf-gmplsextensions-12.txt.

[35] K. Kompella et al., "IS-IS Extensions in Support of Generalized MPLS". IETF, internet draft, RFC Ed Queue. Em fase de desenvolvimento, outubro de 2003. Disponivel em www.ietf.org/internet-drafts/draft-ietf-isis-gmplsextensions-19.txt.

[36] L. Berger et al, "Generalized MPLS-Signaling Functional Description”. IETF, RFC 3471, janeiro de 2003. Disponível em www.ietf.org.

[37] L. Berger et al., "Generalized MPLS Signaling-RSVP-TE Extensions". IETF, RFC 3473, janeiro de 2003. Disponível em www.ietf.org.

[38] P. Ashwood-Smith et al, "Generalized MPLS SignalingCR-LDP Extensions". IETF, RFC 3472, janeiro de 2003. Disponível em www.ietf.org.

[39] D. Papadimitriou D, Z. Lin e D. Pendarakis, "Generalized MPLS (GMPLS) RSVP-TE Usage and Extension for Automatically Switched Optical Network (ASON)". IETF, RFC 3474, março de 2003. Disponível em www.ietf.org.

[40] O. Aboul-Magd, "CR-LDP Extensions for ASON". IETF RFC 3475, março de 2003. Disponível em www.ietf.org.

Eduardo José Aloia graduou-se em Engenharia Elétrica (1989) e obteve o título de Mestre em Engenharia Elétrica (2003) na EESCUSP. Trabalhou como engenheiro de projetos de redes em operadora de telefonia e, atualmente, é aluno de doutorado do curso de pós-graduação em engenharia elétrica da EESC-USP. Suas áreas de pesquisas incluem redes ópticas e GMPLS.

Amílcar Careli César graduou-se em engenharia elétrica na EESC-USP (1976), obteve os títulos de Mestre (1982) e Doutor (1990) em engenharia elétrica na FEEC-UNICAMP, e de LivreDocente em microondas e comunicações ópticas ( 1998) na EESCUSP. Atualmente, exerce as funções de professor associado no Departamento de Engenharia Elétrica da EESC-USP. Participa do Projeto GIGA-CPqD/FINEP/FUNTTEL, como coordenador de subprojeto, e do Projeto Tidia-Kyatera da FAPESP. Suas áreas de pesquisas compreendem a optoeletrônica, microondas e comunicações ópticas. O professor César é membro da Sociedade Brasileira de Telecomunicações--SBrT e da Sociedade Brasileira de Microondas e Optoeletrônica-SBMO.

Murilo Araujo Romero é graduado em Engenharia Elétrica, ênfase em Telecomunicações, pela Pontifícia Universidade Católica do Rio de Janeiro (PUC-Rio) em 1988. Obteve os títulos de Mestre em Ciências (CETUC-PUC/Rio) e Ph.D. (Drexel University, Filadélfia, EUA) em 1991 e 1995, respectivamente. É atualmente professor associado junto ao Departamento de Engenharia Elétrica da Universidade de São Paulo, campus de São Carlos, onde atua no estudo de sistemas e dispositivos para comunicações ópticas. O professor Romero é bolsista de produtividade em pesquisa do $\mathrm{CNPq}$, nível 1 . 
APÊNDICE: TABELAS DE RECOMENDAÇÕES DO ITU-T

\begin{tabular}{|c|c|c|c|}
\hline TóPICO & $\begin{array}{l}\text { RECOMEN- } \\
\text { DAÇÃO }\end{array}$ & TÍTULO & SITUAÇÃOO ${ }^{(*)}$ \\
\hline $\begin{array}{l}\text { Estruturas e } \\
\text { Mapeamento }\end{array}$ & G.709 & Interface for the optical transport network (OTN) & $\begin{array}{l}\text { PUB fev } 2001 \\
\text { REV mar } 2003\end{array}$ \\
\hline $\begin{array}{l}\text { Características } \\
\text { Funcionais }\end{array}$ & G.798 & $\begin{array}{l}\text { Characteristics of optical transport network (OTN) equipment } \\
\text { functional blocks }\end{array}$ & $\begin{array}{l}\text { PUB jan } 2002 \\
\text { REV jun } 2004\end{array}$ \\
\hline $\begin{array}{l}\text { Desempenho de } \\
\text { jitter e wander }\end{array}$ & G.8251 & $\begin{array}{l}\text { (G.otnjit) The control of jitter and wander within the optical } \\
\text { transport network (OTN) }\end{array}$ & $\begin{array}{l}\text { PUB nov } 2001 \\
\text { REV jun } 2002 \\
\end{array}$ \\
\hline \multirow{6}{*}{$\begin{array}{l}\text { Aspectos da } \\
\text { camada física }\end{array}$} & G.694.1 & Spectral grids for WDM applications: DWDM frequency grid & PUB jun 2002 \\
\hline & G.694.2 & Spectral grids for WDM applications: CWDM wavelength grid & PUB jun 2002 \\
\hline & G.664 & $\begin{array}{l}\text { Optical Safety Procedures and Requirements for Optical Transport } \\
\text { Subsystems }\end{array}$ & $\begin{array}{l}\text { PUB jul } 1999 \\
\text { REV mar } 2003 \\
\end{array}$ \\
\hline & G.959.1 & Optical Transport Networking Physical Layer Interfaces & \begin{tabular}{|l|} 
PUB fev 2001 \\
REV dez 2003 \\
\end{tabular} \\
\hline & G.693 & Optical interfaces for intra-office systems & $\begin{array}{l}\text { PUB nov } 2001 \\
\text { REV jan } 2005 \\
\end{array}$ \\
\hline & G Suppl. 39 & Optical system design and engineering considerations & PUB out 2003 \\
\hline \multirow{6}{*}{ Fibras ópticas } & G.651 & $\begin{array}{l}\text { Characteristics of a } 50 / 125 \mu \mathrm{m} \text { multimode graded index optical fiber } \\
\text { cable }\end{array}$ & PUB fev 1998 \\
\hline & G.652 & Characteristics of a single-mode optical fiber cable & $\begin{array}{l}\text { PUB out } 2000 \\
\text { REV mar } 2003\end{array}$ \\
\hline & G.653 & Characteristics of a dispersion-shifted single mode optical fiber cable & \begin{tabular}{|l|} 
PUB out 2000 \\
REV dez 2003 \\
\end{tabular} \\
\hline & G.654 & Characteristics of a cut-off shifted single-mode fiber cable & $\begin{array}{l}\text { PUB jun } 2002 \\
\text { REV jun } 2004 \\
\end{array}$ \\
\hline & G.655 & $\begin{array}{l}\text { Characteristics of a non-zero dispersion shifted single-mode optical } \\
\text { fiber cable }\end{array}$ & $\begin{array}{l}\text { PUB out } 2000 \\
\text { REV mar } 2003\end{array}$ \\
\hline & G.656 & $\begin{array}{l}\text { Characteristics of a fibre and cable with non-zero dispersion for } \\
\text { wideband transport }\end{array}$ & PUB jun 2004 \\
\hline \multirow{4}{*}{$\begin{array}{l}\text { Componentes e } \\
\text { subsistemas }\end{array}$} & G.661 & $\begin{array}{l}\text { Definition and test methods for the relevant generic parameters of } \\
\text { optical amplifier devices and subsystems }\end{array}$ & PUB out 1998 \\
\hline & G.662 & $\begin{array}{l}\text { Generic characteristics of optical fiber amplifier devices and } \\
\text { subsystems }\end{array}$ & PUB out 1998 \\
\hline & G.663 & $\begin{array}{l}\text { Application related aspects of optical fiber amplifier devices and } \\
\text { sub-systems }\end{array}$ & PUB abr 2000 \\
\hline & G.671 & Transmission characteristics of optical components and subsystems & $\begin{array}{l}\text { PUB jun } 2002 \\
\text { REV jan } 2005\end{array}$ \\
\hline
\end{tabular}

Tabela A.1. Recomendações que especificam a rede de transporte óptica (ASON) padronizada pelo ITU-T: Plano de transporte. ${ }^{(*)}$ Data de publicação (PUB); revisão (REV); previsão (PRE).

\begin{tabular}{|c|c|l|l|}
\hline TÓPICO & RECOMENDAÇÃO & \multicolumn{1}{|c|}{ TÍTULO } & SITUAÇÃO $^{(*)}$ \\
\hline \multirow{4}{*}{$\begin{array}{c}\text { Especificações } \\
\text { para o plano } \\
\text { de controle }\end{array}$} & G.7713 & Generalized Call and Connection Management & PUB dez 2001 \\
\cline { 2 - 4 } & G.7713.1 & $\begin{array}{l}\text { Distributed Call and Connection Management-PNNI } \\
\text { Implementation }\end{array}$ & PUB mar 2003 \\
\cline { 2 - 4 } & G.7713.3 & $\begin{array}{l}\text { Distributed Call and Connection Management-GMPLS RSVP- } \\
\text { TE Implementation }\end{array}$ & PUB mar 2003 \\
\cline { 2 - 4 } & Gistributed Call and Connection Management-GMPLS CR- & PUB mar 2003 \\
\cline { 2 - 4 } & GDP Implementation & Generalized automatic discovery techniques & PUB out 2001 \\
\cline { 2 - 5 } & G.7714.1 & Protocol for Automatic Discovery in SDH and OTN Networks & PUB abr 2004 \\
\cline { 2 - 5 } & G.7715.1 & $\begin{array}{l}\text { Architecture and requirements for routing in automatically } \\
\text { switched optical networks }\end{array}$ & PUB jun 2002 \\
\hline
\end{tabular}

Tabela A.2. Recomendações que especificam a rede de transporte óptica (ASON) padronizada pelo ITU-T: Plano de controle. ${ }^{(*)}$ Data de publicação (PUB); revisão (REV); previsão (PRE). 
is

\begin{tabular}{|c|c|c|c|}
\hline TÓPICO & RECOMENDAÇÃO & TítuLO & 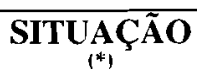 \\
\hline \multirow{5}{*}{$\begin{array}{l}\text { Aspectos de } \\
\text { Gerenciamento }\end{array}$} & G.874 & Management aspects of the optical transport network element & $\begin{array}{l}\text { PUB nov } \\
2001\end{array}$ \\
\hline & G.874.1 & $\begin{array}{l}\text { Optical Transport Network (OTN) Protocol-Neutral Management } \\
\text { Information Model For The Network Element View }\end{array}$ & $\begin{array}{l}\text { PUB jan } \\
2002\end{array}$ \\
\hline & G 7718 & Framework for ASON management & $\begin{array}{l}\text { PUB fev } \\
2005\end{array}$ \\
\hline & G.875 & OTN Management Information Model - CMIP Implementation & PRE 2006 \\
\hline & G.876 & OTN Management Information Model - SNMP Implementation & PRE 2006 \\
\hline
\end{tabular}

Tabela A.3. Recomendações que especificam a rede de transporte óptica (ASON) padronizada pelo ITU-T: Plano de gerenciamento. ${ }^{\left({ }^{*}\right\}}$ Data de publicação (PUB); revisão (REV); previsão (PRE).

\begin{tabular}{|l|c|l|l|}
\hline \multicolumn{1}{|c|}{ TÓPICO } & RECOMENDAÇÃO & \multicolumn{1}{c|}{ TÍTULO } & SITUAÇÃO ${ }^{(*)}$ \\
\hline $\begin{array}{l}\text { Framework para as } \\
\text { recomendações }\end{array}$ & G.871 & $\begin{array}{l}\text { Framework for Optical Transport Network } \\
\text { Recommendations }\end{array}$ & PUB out 2000 \\
\hline & G.872 & Architecture of Optical Transport Networks & $\begin{array}{l}\text { PUB fev 1999; } \\
\text { REV nov 2001 }\end{array}$ \\
\cline { 2 - 5 } $\begin{array}{l}\text { Arquitetura e } \\
\text { Plano de controle }\end{array}$ & G.807 & $\begin{array}{l}\text { Requirements for the Automatic Switched Transport } \\
\text { Network (ASTN) }\end{array}$ & PUB jul 2001 \\
\cline { 2 - 5 } & G.8080 8081 & $\begin{array}{l}\text { Architecture for the Automatically Switched Optical } \\
\text { Networks }\end{array}$ & PUB nov 2001 \\
\hline $\begin{array}{l}\text { Desempenho de erro } \\
\text { Optical Networks (ASON) }\end{array}$ & G.8201 & $\begin{array}{l}\text { Error performance parameters and objectives for multi- } \\
\text { operator international paths within the Optical } \\
\text { Transport Network (OTN) }\end{array}$ & PUB set 2003 \\
\hline $\begin{array}{l}\text { Especificação da rede } \\
\text { de transporte de } \\
\text { dados (Data } \\
\begin{array}{l}\text { Communication } \\
\text { Network-DCN) }\end{array}\end{array}$ & G.7712 & $\begin{array}{l}\text { Architecture and specification of data communication } \\
\text { network }\end{array}$ & PUB mar 2003 \\
\hline
\end{tabular}

Tabela A.4. Recomendações que especificam a rede de transporte óptica (ASON) padronizada pelo ITU-T: Arquitetura de redes ópticas. ${ }^{(*)}$ Data de publicação (PUB); revisão (REV); previsão (PRE). 